\title{
Risk factors for suicide in depression in Finland: first-hospitalized patients followed up to 24 years
}

Aaltonen KI, Isometsä E, Sund R, Pirkola S. Risk factors for suicide in depression in Finland: first-hospitalized patients followed up to 24 years

Objective: To examine longitudinally risk factors for suicide in depression, and gender differences in risk factors and suicide methods. Method: We linked data from (i) The Finnish Hospital Discharge Register, (ii) the Census Register of Statistics Finland, and (iii) Statistics Finland's register on causes of deaths. All 56826 first-hospitalized patients (25 188 men, 31638 women) in Finland in 1991-2011 with a principal diagnosis of depressive disorder were followed up until death (2587 suicides) or end of the year 2014 (maximum 24 years).

Results: Clinical characteristics (severe depression adjusted hazard ratio [AHR] 1.19 [95\% CI 1.08-1.30]; psychotic depression AHR 1.45 [1.301.62]; and comorbid alcohol dependence AHR 1.26 [1.13-1.41]), male gender (AHR 2.07 [1.91-2.24]), higher socioeconomic status and living alone at first hospitalization were long-term predictors of suicide deaths. Highest risk was associated with previous suicide attempts (cumulative probability 15.4\% [13.7-17.3\%] in men, 8.5\% [7.3-9.7\%] in women). Gender differences in risk factors were modest, but in lethal methods prominent.

Conclusion: Sociodemographic and clinical characteristics at first hospitalization predict suicide in the long term. Inpatients with previous suicide attempts constitute a high-risk group. Despite some gender differences in risk factors, those in lethal methods may better explain gender disparity in risk.

\section{K. I. Aaltonen ${ }^{1,2}$ (iD, E. Isometsä ${ }^{1}$ (D) R. Sund ${ }^{3,4}$, S. Pirkola ${ }^{2,5}$}

${ }^{1}$ Department of Psychiatry, University of Helsinki, Helsinki, ${ }^{2}$ Mental Health Unit, Department of Public Health Solutions, National Institute for Health and Welfare, Helsinki, ${ }^{3}$ Faculty of Social Sciences, Centre for Research Methods, University of Helsinki, Helsinki,

${ }^{4}$ Institute of Clinical Medicine, University of Eastern Finland, Kuopio, and ${ }^{5}$ Faculty of Social Sciences and Pirkanmaa Hospital District, University of Tampere, Tampere, Finland

Key words: depression; suicide; cohort studies; risk factors; gender differences

Erkki Isometsä, Department of Psychiatry, University of Helsinki, P.O. Box 22 (Välskärinkatu 12 A), Fl-00014, Helsinki, Finland. E-mail: erkki.isometsa@hus.fi

Accepted for publication November 22, 2018

\section{Significant outcomes}

- This national study of risk factors for suicide in depression is the largest cohort study published thus far in terms of sample size and number of suicide deaths. Of 13 risk factors, male sex, previous suicide attempts, severe or psychotic depression, comorbid alcohol dependence, higher family income, and higher education predicted future suicide.

- During the long-term follow-up, a substantial proportion of patients hospitalized for depression with a history of suicide attempt eventually died by suicide.

- Some gender differences in risk factors for suicide in depression were found to exist. However, there are marked gender differences in lethal methods used, which more likely explain the gender disparity in risk of suicide.

\section{Limitations}

- The cohort comprised first-hospitalized psychiatric inpatients. Generalizability of findings pertaining to risk factors among psychiatric outpatients or primary care patients remains to be confirmed.

- Some risk factors remained uninvestigated in the study.

- The study is based on clinical diagnoses. Diagnostic imprecisions likely exist in assessment of severity of depression, in poor recognition of delusional hopelessness of psychotic depression, and missed diagnoses of comorbid alcohol dependence. 


\section{Risk factors for suicide in depression}

\section{Introduction}

Of all individuals dying by suicide, about one-half have suffered from depressive disorders (1) and nearly one-half have a history of psychiatric hospitalization $(2,3)$. Hospitalized patients with affective disorders $(4,5)$ or severe depression $(6-8)$ have high risk of suicide. However, prospective evidence on risk factors for suicide deaths in depression is very limited (9). The same applies to previously hospitalized patients (10). Low incidence of suicides in the shortterm necessitates very large sample sizes and extended follow-ups to reliably estimate risk factors for suicide in cohort studies. Consequently, most suicide research has focused on proxy outcomes of suicide attempts or ideation, leaving uncertainty in the generalizability of the findings (11).

According to a systematic review of 29 putative risk factors (9), suicide deaths in depression are associated with male gender, family history of psychiatric disorder, previous suicide attempt, depression severity, hopelessness, comorbid anxiety and personality disorders, and substance use disorders. However, the evidence is mostly based on a limited number of small case-control or cohort studies, and considerable uncertainty persists regarding even clinically relevant risk factors such as psychotic features (9). For several reasons, attempts to identify high-risk groups have thus far been ineffectual (10). Longitudinal large-scale studies on risk factors for suicide in depression are needed to aid clinical evaluations, treatment planning, and preventive efforts (9).

The gender disparity of suicide risk among subjects with depression (9) constitutes a central finding calling for scientific explanation. The excess mortality in men might be explained by gender differences in comorbid mental disorders (12), suicidal intent $(13,14)$, impulsive-aggressive traits $(15)$, or choice of lethal methods (13). Gender differences in risk factors for suicide attempts in depression may exist (16). However, as reliable longitudinal evaluation of gender differences in risk factors necessitates substantially larger cohorts than mere investigation of risk factors overall, gender differences in risk factors for suicide deaths among patients with depression have not been investigated.

The data of the study are based on a Finnish population-based cohort of all first-hospitalized patients $(N=56826)$ due to depression in 19912011 who were followed up after discharge until the end of the year 2014 (maximum 24 years). In our previous work (8), we have reported the overall cumulative risk of suicide being higher in men than in women and that the risk of suicide declined nationwide to one-half (hazard ratio 0.49 ) between those hospitalized during years 1991-1995 and those during years 2006-2011.

Aims of the study

In this Finnish national register-based study, we investigate altogether 13 plausible risk factors for suicide after first hospitalization for depression over an extended follow-up period of 24 years at most. We examine gender differences in risk factors and methods for suicide.

\section{Material and methods}

Study design, setting, and population

This study stems from the MERTTU research project (17). The database merges data from three national sources: (i) the Finnish Hospital Discharge Register on all inpatient treatments, (ii) the census register of Statistics Finland on sociodemographic variables, and (iii) the Statistics Finland's register on causes of death. The Finnish personal identity codes link complete data at the individual level.

We identified all $\geq 18$-year-old patients with first lifetime admission to a psychiatric hospital or a psychiatric ward of a general hospital for depressive disorder (principal diagnosis) in 1991-2011. The overall annual population rate of psychiatric inpatient care in Finland has varied between 5.3 per 1000 inhabitants and 6.3 per 1000 inhabitants in 1994-2011 (18).

A total of 56826 subjects (25 188 men, 31638 women) were followed on the registers until death by suicide or other cause or the end of the year 2014 (length of follow-up 628514 person-years, mean 11.1 years, median 10.7 years, maximum 24 years). The Ethics Committee of the National Institute for Health and Welfare approved the study design.

\section{Finnish Hospital Discharge Register}

The Finnish Hospital Discharge Register (FHDR) contains information on all psychiatric and somatic hospitalizations since 1969. In Finland, psychiatric secondary services receive funding through tax revenue, and there are no private psychiatric hospitals. Since 1987, national guidelines have stipulated use of operationalized criteria for clinical psychiatric diagnoses. The Finnish ICD-9 diagnoses (19) used in 1987-1995 were based on the DSM-III-R criteria and the ICD-10 diagnoses since then on the Diagnostic Criteria for Research (DCR). The accuracy of mental health diagnoses in the FHDR is good (20). 


\section{Aaltonen et al.}

We identified from the database a cohort of patients having at least one admission with a psychiatric diagnosis in 1987-2011. Due to the availability of sociodemographic information since 1990, the study period starts in 1991. We identified baseline (first) hospitalizations for depressive disorder as a principal diagnosis in 1991-2011 with the Finnish ICD-9 codes (19) 2961A-G and 2968A and ICD-10 codes F32-33. We obtained for this cohort all hospitalizations with psychiatric diagnoses in 1980-2011 and excluded patients with any previous psychiatric hospitalizations in 1980-1990 (including hospitalizations before the age of 18 years). Excluded were also patients with baseline comorbid diagnosis of principal psychotic disorder (ICD-9 codes 2951-2953A-F, 2954A, 2956A-F, 2957A， 2959A-F, 2971A， 2973A, 2988A, 2989X, 3012C; ICD-10 codes F20-29) or bipolar disorder (postdischarge suicide risk in bipolar disorder has been previously reported (21)), or who died by suicide during baseline hospitalization. We extracted data on comorbid diagnoses of alcohol dependence (ICD-9 code 3039X; ICD-10 code F10.2), date of admission, and discharge. Since 1994, the FHDR provides information on the overall functioning at discharge (Global Assessment Scale [GAS] value recorded by attending doctor) and admission status (voluntary, involuntary). In Finland, a doctor is authorized to refer a person involuntarily for evaluation in a psychiatric hospital when three conditions are met: (i) the patient is mentally ill (i.e. psychotic), (ii) he or she needs treatment for a mental illness, which if left untreated would become considerably worse or severely endanger the health or safety of the patient or others, and (iii) all other mental health services are considered inapplicable or inadequate.

\section{Suicide deaths and previous attempts}

Finland has high medicolegal autopsy rates (about $16-24 \%$ of all deaths in 1991-2014) $(22,23)$. A forensic pathologist performs a medicolegal autopsy in almost every case of unnatural death and determines virtually all suicides (leaving few undetermined deaths in a ratio of less than 1 to 10 relative to suicides) (22). We retrieved information on the dates and causes of death for all cases and then identified suicides (ICD-9 codes E950-E959; ICD-10 codes X60-X84, Y87.0, Z91.5).

We identified previous suicide attempts (codes as above) from somatic and psychiatric records in the FHDR. Previous suicide attempts were classified in one of two non-overlapping categories: (i) a suicide attempt at admission (either somatic hospitalization due to a suicide attempt which interminably continued thereafter as baseline psychiatric hospitalization or suicide attempts recorded in psychiatric discharge records by attending psychiatrist [latter includes also suicide attempts treated in emergency department and non-medical attention-requiring attempts either preceding admission or attempted during the baseline hospitalization due to identical coding in the register]) or (ii) suicide attempts during the 4 years prior to baseline (all had resulted in hospital admission, excludes emergency department visits). The 4-year time-frame was determined by establishing equal time-at-risk period for each patient (data available since 1987). Neither data on preexisting psychiatric diagnoses at the time of the past attempts nor evaluation of suicidal intent (ICD codes include suicide attempts and other intentional self-harm) was available in the registers. However, patients who self-harm without suicidal intent are generally treated in psychiatric outpatient setting. Because suicide attempts in the data have either necessitated somatic inpatient treatment or contributed to baseline psychiatric admissions, we assume that some degree of suicidal intent has been present.

\section{Sociodemographics}

The census register of Statistics Finland provided data from the final day of the previous year for baseline hospitalization on the following six variables: (i) marital status (categorized as single, married, divorced, and widowed; includes same-sex partners since 2002), (ii) habitation (living with family or alone), (iii) having children $\leq 7$ years of age (for patients living with the family), (iv) family income (relative within the cohort, divided into thirds), (v) educational level (basic, upper secondary, and tertiary), and (vi) employment status (at work, unemployed, student, retired, conscript or conscientious objector, unemployment pension, or not in labor force for other reason).

\section{Statistical analyses}

The DSM-III-R-based diagnoses from the ICD-9 era were converted to corresponding ICD-10-DCR diagnoses. The study diagnoses were then grouped into three mutually exclusive subcategories of severity: (i) reference (henceforth 'moderate') category included mild, moderate, full or partial remissions, other specified, and unspecified types (ICD-9 codes 2961A-C, 2961F-G, 2968A; ICD-10 codes F32.0*, 


\section{Risk factors for suicide in depression}

F32.1*, F32.8, F32.9, F33.0*, F33.1*, F33.4, F33.8, F33.9), (ii) severe depressive disorder without psychotic features or symptoms (ICD-9 code 2961D; ICD-10 codes F32.2, F33.2), and (iii) severe depressive disorder with psychotic features or symptoms (ICD-9 code 2961E; ICD-10 codes F32.3, F33.3).

We followed the patients using register data from the day of discharge to death by suicide or other cause, or December 31, 2014, whichever occurred first. Other causes of death were considered as competing risks for suicides. Observations in which the end of follow-up period was reached were treated as censored. During the naturalistic observation period, re-hospitalizations were possible and suicide deaths include all suicides irrespective of the place of death. Because of risk of survival bias, we ignored diagnostic conversions to a principal psychotic disorder or bipolar disorder (only diagnoses of living patients can change).

We used the competing survival analyses to derive the cumulative probability of dying by suicide. Cause-specific Cox proportional hazard regression models were used to provide unadjusted (sex, age, baseline year only) and adjusted hazard ratio (AHR) estimates for the association between covariates and risk of suicide. Separate models were estimated for all and for men and women. Statistical significances of gender interactions were estimated by including interaction term for one variable at a time to the model. The selection of variables to the multivariate models was made by a priori hypotheses. Decisions were also based on an aim to limit interdependence of included variables or their susceptibility to changes during the long-term follow-up. We chose variables indicating sociodemographic status being family income, education level, and living alone. Baseline year was used as a stratification variable in the Cox models. According to observations based on Schoenfeld residuals, the proportional hazards assumption was met. We used the software packages of $\mathrm{R}$ (www. r-project-org), and Survo (www.survo.fi).

\section{Results}

Patient characteristics at baseline hospitalization are presented in the Table S1. The cumulative probability of dying by suicide was $7.7 \% \quad(95 \%$ CI $7.2-8.2 \%)$ in men and $3.9 \%(95 \%$ CI $3.6-$ $4.3 \%$ ) in women (overall $5.6 \%(95 \%$ CI $5.3-$ $5.9 \%)$ ). The temporal patterns of risk of suicide in men and women are presented in Fig. 1. The mean age at the time of suicide was 46.2 (SD 13.9) in men and 48.4 (SD 14.7) in women (overall 47.0; SD 14.3).

\section{Predictors of suicide}

The unadjusted (age only) hazard ratios for suicide are provided in the Table S2. Suicide deaths were associated with numerous variables including gender, severity of depression, alcohol dependence, previous suicide attempts, education level, family income, living alone, marital status, having small children in the family, employment status, and GAS score.

The multivariable-adjusted models for proportional hazards of suicide (contribution of each variable adjusted for the confounding effects of other variables) are presented for all patients and for men and women separately in adjacent columns of Table 1. Men showed an AHR of 2.07 (95\% CI 1.91-2.24; $P<0.0001)$ for suicide. Severity of depression showed an exposure-response gradient (severe depression without psychotic symptoms AHR 1.19; 95\% CI 1.08-1.30; $P=0.0003$; psychotic depression AHR 1.45; $95 \%$ CI $1.30-1.62 ; P<0.0001)$ and comorbid alcohol dependence an AHR of 1.26 (95\% CI 1.13-1.41; $P<0.0001)$ for risk of suicide. A previous suicide attempt, either at baseline or during the previous 4 years, elevated the risk for suicide most by over twofold. Of the sociodemographic factors at baseline, the risk of suicide was increased by living alone, higher level of education, and high family income.

Of those with previous suicide attempts, the overall cumulative probability of suicide was $15.4 \%(95 \%$ CI $13.7-17.3 \%)$ in men (suicide attempts at admission $15.5 \%$; 95\% CI $13.2-$

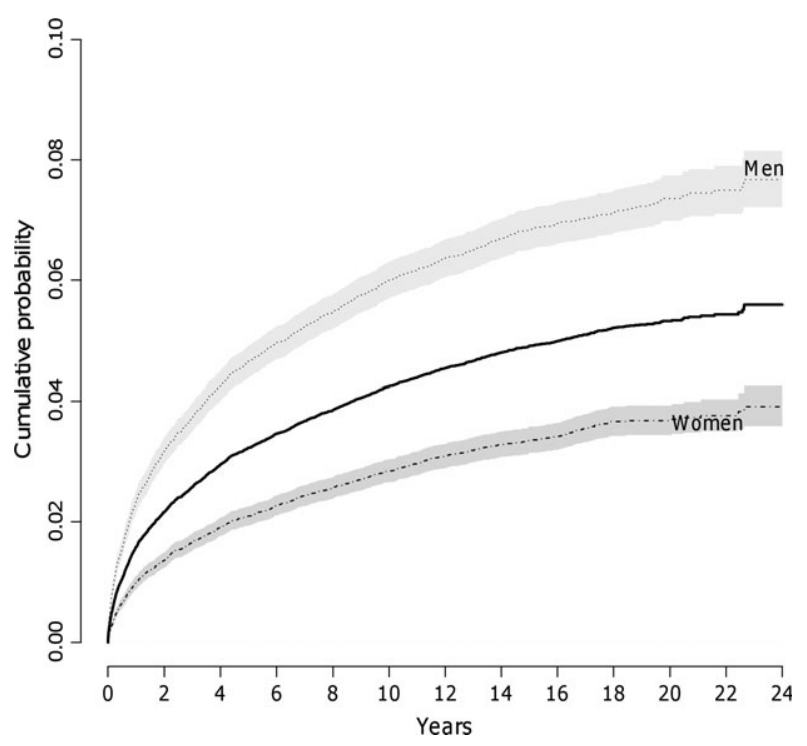

Fig. 1. Cumulative probability of dying by suicide in men and women after first lifetime psychiatric hospitalization for depression followed up to a maximum of 24 years. 


\section{Aaltonen et al.}

$17.9 \%$; and during the previous 4 years $16.0 \%$; $95 \%$ CI $13.5-18.6 \%)$, and $8.5 \%$ (95\% CI $7.3-$ $9.7 \%$ ) in women (suicide attempts at admission $8.4 \%$; 95\% CI $6.9-10.0 \%$; and during the previous 4 years $8.8 \% ; 95 \%$ CI $7.1-10.7 \%$ ). Of patients with a previous suicide attempt, $9.7 \%$ of men and $5.0 \%$ of women died by suicide within 5 years of discharge. The temporal patterns of cumulative probability of suicide in men and women with previous suicide attempts are presented in Fig. 2. At baseline, the patients who had a history of a previous suicide attempt within past 4 years were about 5 years younger (mean age 39.5 years [SD 13.3] in men and 39.5 years [SD 15.1] in women) compared to the total cohort. Among these patients, the most common methods for the first attempted suicide were intoxication $(90.7 \%)$ and cutting $(3.5 \%)$.

Differences in predictors and methods of suicide in men and women

Some risk factors for suicide were stronger in one gender. First, a diagnosis of comorbid alcohol dependence increased the risk of suicide proportionally more in women than in men (statistically strongest gender interaction term, $P=0.002$ ). Second, tertiary level education specifically increased significantly the risk of suicide in women but not in

men (interaction $P=0.01$ ). Third, having young children in the family increased the risk of suicide in men but not in women (interaction $P=0.03$ ). No other statistically significant gender differences were detected as a whole, except for admission type (interaction $P=0.11$ ), involuntary admission seemed to increase the risk for suicide more in women than men (pairwise interaction $P=0.046$ ). Previous suicide attempts and family income showed least evidence for gender differences.

The methods of suicide deaths in men and women are presented in Table 2. Relative to women, men died more often by self-poisoning with gases; hanging, strangulation, or suffocation; and use of firearms. Women died more often than men by drowning or self-poisoning.

\section{Discussion}

This national study of risk factors for suicide in depression among 56826 first-time hospitalized patients, followed up to 24 years, is the largest of its kind to date. Unlike in any previous study, the data enabled investigation of gender differences in altogether 13 risk factors. Overall, baseline predictors of suicide were male gender, previous suicide attempts, severe or psychotic depression, comorbid alcohol dependence, higher socioeconomic status

Table 1. Adjusted hazard ratios for suicide after first lifetime psychiatric hospitalization for depression followed for 628514 person-years $\dagger$

\begin{tabular}{|c|c|c|c|c|c|c|c|c|c|}
\hline & \multicolumn{3}{|c|}{ All $(N=56$ 826) } & \multicolumn{3}{|c|}{ Men ( $n=25$ 188) } & \multicolumn{3}{|c|}{ Women ( $n=31$ 638) } \\
\hline & $H R$ & $95 \% \mathrm{Cl}$ & $P$ & $H R$ & $95 \% \mathrm{Cl}$ & $P$ & $\mathrm{HR}$ & $95 \% \mathrm{Cl}$ & $P$ \\
\hline Men & 2.069 & $1.908-2.243$ & $<0.0001$ & - & & & - & & \\
\hline \multicolumn{10}{|l|}{ Depression severity } \\
\hline Moderate $\ddagger$ & 1.0 & & & 1.0 & & & 1.0 & & \\
\hline Severe without psychotic symptoms & 1.188 & $1.083-1.303$ & 0.0003 & 1.230 & $1.095-1.382$ & 0.0005 & 1.115 & $0.956-1.299$ & n.s. \\
\hline Alcohol dependence ${ }^{* *}$ & 1.261 & $1.129-1.409$ & $<0.0001$ & 1.147 & $1.008-1.306$ & 0.038 & 1.647 & $1.338-2.026$ & $<0.0001$ \\
\hline \multicolumn{10}{|l|}{ Suicide attempt $\S$} \\
\hline At admission & 2.110 & $1.862-2.391$ & $<0.0001$ & 2.000 & $1.700-2.355$ & $<0.0001$ & 2.248 & $1.849-2.733$ & $<0.0001$ \\
\hline During previous 4 years & 2.111 & $1.845-2.415$ & $<0.0001$ & 2.116 & $1.783-2.510$ & $<0.0001$ & 2.083 & $1.673-2.592$ & $<0.0001$ \\
\hline \multicolumn{10}{|l|}{ Education* } \\
\hline Basic & 1.0 & & & 1.0 & & & 1.0 & & \\
\hline Upper secondary & 1.147 & $1.049-1.253$ & 0.003 & 1.128 & $1.010-1.260$ & 0.032 & 1.177 & $1.013-1.369$ & 0.034 \\
\hline \multicolumn{10}{|l|}{ Income } \\
\hline Lowest third & 1.0 & & & 1.0 & & & 1.0 & & \\
\hline Middle third & 1.065 & $0.961-1.180$ & n.s. & 1.060 & $0.929-1.210$ & n.s. & 1.059 & $0.899-1.248$ & n.s. \\
\hline Highest third & 1.191 & $1.068-1.329$ & 0.002 & 1.169 & $1.018-1.343$ & 0.027 & 1.207 & $1.011-1.442$ & 0.038 \\
\hline Living alone & 1.124 & $1.029-1.227$ & 0.009 & 1.165 & $1.044-1.301$ & 0.007 & 1.069 & $0.923-1.238$ & n.s. \\
\hline
\end{tabular}

$\mathrm{Cl}$, confidence interval; HR, hazard ratio; n.s., not significant; $P$, $P$-value.

Statistically significant $P$-values $(P<0.05)$ are bolded.

${ }^{*}$ Statistically significant interaction between $\operatorname{sex}, P<0.05$

** Statistically significant interaction between sex, $P<0.01$.

$\uparrow$ Adjusted for each other and age, baseline admission year was used as a stratification variable.

\$Includes mild to moderate, full, and partial remissions, other specified and unspecified types of depression.

$\S$ Non-overlapping categories, an individual may have both.

qLowest level tertiary education or higher. 

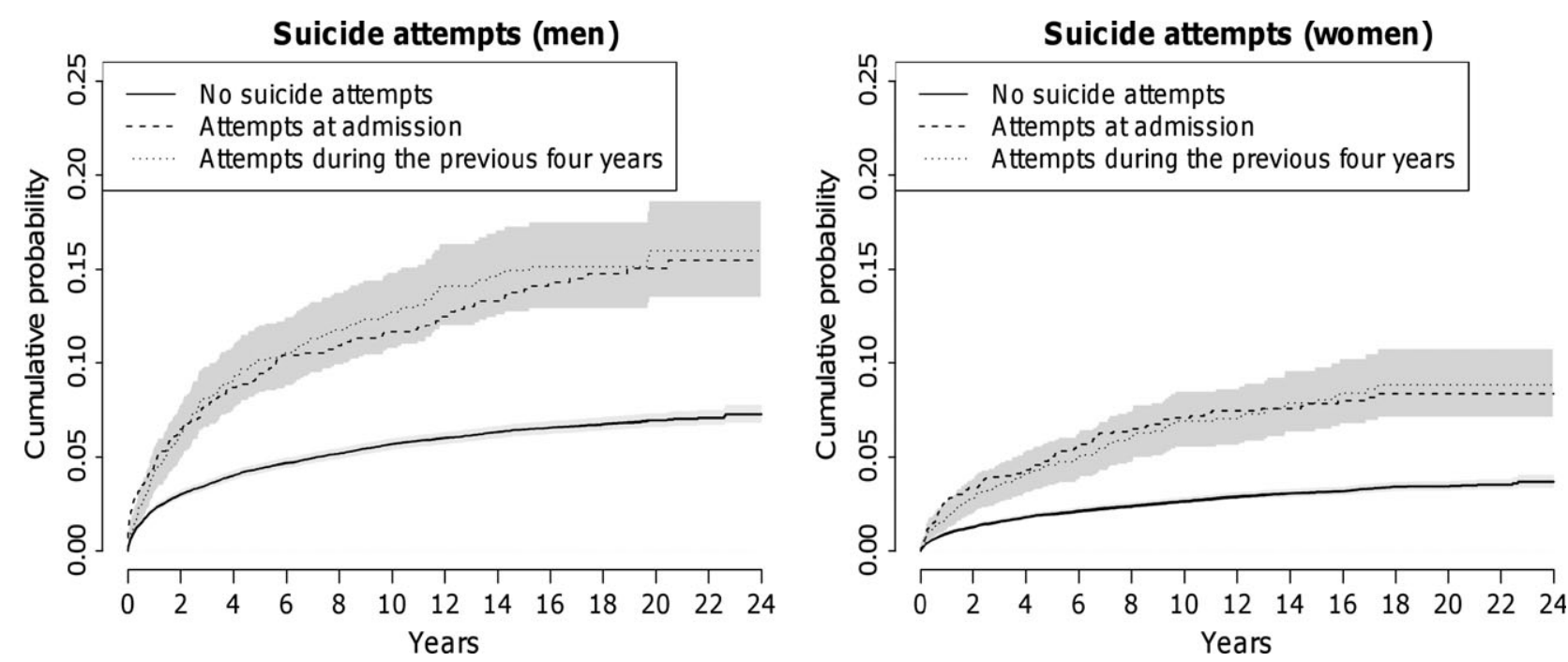

Fig. 2. Cumulative probability of dying by suicide after a suicide attempt, either during the previous 4 years or at the baseline admission, in men and women after first lifetime psychiatric hospitalization for depression.

and living alone. Men with previous suicide attempts had very high risk of suicide death. Gender differences in risk factors were found in comorbid alcohol dependence, tertiary level education, and having young children in the family. The order of magnitude of these differences was, however, limited compared with the large gender

Table 2. Methods of suicide in men and women $(n=2587)$ dying by suicide after first lifetime psychiatric hospitalization for depression followed for a maximum of 24 years

\begin{tabular}{|c|c|c|c|c|}
\hline \multirow[b]{2}{*}{ Method of suicide } & \multicolumn{2}{|c|}{$\begin{array}{c}\text { Men } \\
(n=1609)\end{array}$} & \multicolumn{2}{|c|}{$\begin{array}{l}\text { Women } \\
(n=978)\end{array}$} \\
\hline & $n$ & $\%$ & $n$ & $\%$ \\
\hline Gassing* & 74 & 4.6 & 8 & 0.8 \\
\hline Hanging, strangulation, or suffocation* & 540 & 33.6 & 192 & 19.6 \\
\hline Drowning* & 77 & 4.8 & 115 & 11.8 \\
\hline Firearms and explosives * & 193 & 12.0 & 19 & 1.9 \\
\hline Jumping from a height & 73 & 4.5 & 54 & 5.5 \\
\hline Cutting and piercing & 42 & 2.6 & 15 & 1.5 \\
\hline Self-poisoning ${ }^{*}$ & 475 & 29.5 & 495 & 50.6 \\
\hline Traffic & 105 & 6.5 & 72 & 7.4 \\
\hline Sequelae of intentional self-harm and other methods & 30 & 1.9 & 8 & 0.8 \\
\hline
\end{tabular}

Chi-square 265.7; df 8; $P=<0.0001$.

Gassing includes ICD-9 codes E951*and E952* and ICD-10 code X67.

Hanging, strangulation, or suffocation includes ICD-9 code E953* and ICD-10 code $\mathrm{X} 70$.

Drowning includes ICD-9 code E954* and ICD-10 code X71.

Firearms and explosives include ICD-9 code E955* and ICD-10 codes X72-X75.

Jumping from a height includes ICD-9 code E957* and ICD-10 code X80.

Cutting and piercing include ICD-9 code E956* and ICD-10 code X78.

Self-poisoning includes ICD-9 code E950* and ICD-10 codes X60-X66, X68-X69. Traffic includes ICD-9 codes E959A, E959B, and E959C and ICD-10 codes X81-X82.

Sequelae of intentional self-harm and other methods include ICD-9 code E959X and ICD-10 codes X76, X77, X79, X83-X84, Y870, and Z915.

${ }^{*}$ Relative frequency significantly different between men and women at the 0.0001 level (z-test). difference in risk. We found marked gender differences in lethal methods of suicide.

\section{Predictors of suicide}

We investigated 13 putative risk factors for suicide in depression, including 11 of the 29 examined in the recent meta-analysis (9), plus income, and education level. To our knowledge, this is a wider range than in any previous longitudinal study and enables also examination of adjusted effects. The Hawton et al. (9) meta-analysis included three larger cohorts, of which one was a national sample (24) with an investigation period in 1973-1993 mostly preceding ours and none of these examined more than five risk factors. In our data, predictors were examined in a national sample with 2587 suicides. By comparison, the pooled meta-analytic data (9) on depression severity or alcohol misuse derived from a limited number of small samples, including less than 300 suicides. The other limitations of previous studies include samples of advanced age, only men, or retrospective study designs. The systematic review (10) of risk factors for suicide of discharged general psychiatric inpatients was based on 1544 suicides.

Diagnosed severity of baseline depressive episode in our data shows a dose-response effect on long-term risk of suicide, thereby confirming the previous tentative findings of a smaller, likely under-powered study (25). Severity of depression, when measured more accurately with the Patient Health Questionnaire (PHQ-9) in a large community sample, has been found to predict suicide death over the next month (26). Those results enable generalizability to less severely depressed 


\section{Aaltonen et al.}

outpatient populations, whereas our long-term findings are based on a high number of suicide deaths and involve additionally psychotic depression. Severity of depression has been found to correlate significantly with severity of suicidal ideation, although even among severely depressed patients some have had no suicidal thoughts (27). The role of psychotic symptoms in risk of suicide in depression has been long debated. Our finding of an added influence of diagnosed psychotic depression on risk of suicide substantiates the so far limited evidence base (9), but is contrary to the findings of a recent study (28) applying different study methodologies. According to other studies, baseline severity of depression $(25,29)$ and psychotic features (30) predict future recurrence and persistence of depressive morbidity. Time spent at risk in gradually more severe depressive episodes likely forms a major determinant of suicide risk and provides a meaningful target for prevention (11).

We found alcohol dependence to elevate risk for suicide by $26 \%$ in the adjusted models. This finding is in accordance with the Danish national study (7) and the Hawton et al. (9) meta-analysis but in contrast to the findings of some major studies (10, 24). Given that alcohol use disorders rank as the second most common mental disorder among suicide decedents (1), we believe that depressed inpatients with alcohol use disorders represent a high-risk group needing preventive attention.

Of first-hospitalized patients with depression and previous suicide attempts more than one in seven $(15 \%)$ men and more than one in twelve $(8.5 \%)$ women eventually died by suicide. The risk was highest in the early years but persisted over nearly two decades. The twofold adjusted risk is slightly higher, but comparable with the estimations of a Danish national study (7). The gender difference in risk reflected the higher overall risk among men and we found no gender-modifying effects pertaining to previous suicide attempts among inpatients. Overall, our estimations of suicide risk markedly exceed those of a previous meta-analysis of self-harm patients (31), which pooled data mostly on self-poisonings in primary and secondary care settings. In contrast, our data derive from suicide attempts that had mostly necessitated somatic admission among patients later hospitalized for depression with extended follow-up, which may explain the differences. Individuals who use violent methods have high risk of repetition with fatal outcome particularly in the short term (32). However, the methods, medical severity, or intent of previous suicide attempts were unmodeled in our study. The high suicide risk of first-hospitalized patients with preceding suicide attempts, highlighted particularly in men, is understandable as a proxy for several important risk indicators for suicide, that is, preceding suicide attempts (9), psychiatric hospitalizations (2), highrisk postdischarge periods (33), and early illness course (7). The high proportion $(56-59 \%)$ of suicide attempters dying by their first attempt $(34,35)$ limits, however, the sensitivity as a risk indicator. Nevertheless, psychiatric evaluation should be provided to all who have attempted suicide. Depressive inpatients who have previously attempted suicide form a particular high-risk group warranting intensive attention in psychiatric care.

Of sociodemographic factors, living alone, high relative family income, and higher educational level predicted suicide deaths. Our finding that living alone increases the risk of suicide in depression adds to the previously insufficient evidence base (9). The finding of baseline higher socioeconomic status predicting higher risk of suicide among inpatients is counterintuitive, but not without support (36). Contrasting results are found in communitybased case-control studies $(2,36,37)$, in which suicide deaths have been associated with single marital status, unemployment, sickness-related absence, and low income. However, when in these general population studies the effect of a history of psychiatric hospitalization was controlled for, the associations between socioeconomic status and suicide were often disappeared indicating major role for mental illness. A cohort study as here provides better control for confounding and our finding persisted when controlling for baseline diagnosed severity of depression. However, some residual confounding by illness severity may remain due to complex bidirectional relations between sociodemographic factors and depression (discussed in more detail elsewhere $(36,38))$. Moreover, we modeled baseline information on socioeconomic status prior to hospitalization and the socioeconomic status may have changed considerably at the time of death. Accordingly, another longitudinal study of general psychiatric inpatients (39) showed not only that higher socioeconomic status was associated with higher risk of suicide but also that this higher risk was associated with later loss of income or employment status and ending of an intimate relationship. In summary, our findings of higher education and income at first hospitalization for depression to predict higher future suicide risk are consistent, but the causal pathways remain unclear and require further studies. Possible explanations include that higher socioeconomic status could mark some unmeasured baseline vulnerability, or that depressive inpatients with a higher 
socioeconomic status might have been at greater suicide risk if they later experienced major hardships in living circumstances.

Differences in predictors and methods of suicide in men and women

In women, diagnosed comorbid alcohol dependence elevated the risk of suicide more than in men. Relative risks should not be confused with absolute risks, which among alcohol-dependent women remain lower than the risk among nonalcohol-dependent men. In the Finnish psychological autopsy study in 1987-1988, male decedents with major depression had more often comorbid alcohol disorder and less often a history of psychiatric hospitalization (40). We found that high education level increased the risk for suicide more in women than men, whereas another study (41) found an interaction with male gender and medium-level education. In contrast to previous studies $(2,37,41)$, we found no evidence on protective effects of having young children in the family. Conversely, in men this was statistically a significant risk factor, although marginal in magnitude of effect.

Gender differences in quality, distribution, or potency of underlying risk and protective factors for suicide could partly explain the twofold higher risk among men. In the adjusted analyses, however, the risk associated with male gender remained unaltered. This suggests a role of other factors in explaining the gender disparity in risk. According to the Finnish psychological autopsy studies $(34,42)$, we found that men died more often than women by potentially more lethal suicide methods. Gender differences in methods of suicide are marked, whereas variations in prevalence or potency of risk factors are modest. We cannot exclude the possibility of gender differences in some unmeasured risk factors, but our findings are more consistent with differences in lethality of the suicide methods chosen contributing to this disparity.

\section{Strengths and limitations of the study}

Strengths of the study design include a populationbased cohort of first-hospitalized patients $(N=56$ 826) with depression in 1991-2011 followed for a maximum of 24 years (including 2587 suicides). The large data enable in a single study design longitudinal examination of adjusted effects of numerous risk factors overall, and a comparison between men and women. The study strengths include high-quality national-level register data and complete information on postdischarge suicide mortality based on reliable death investigation procedures (22). The data on methods of suicide and on risk factors demonstrate the magnitude of gender differences in both.

Among the limitations are that structural interview-based diagnostics remain unavailable for large-scale studies. The clinical diagnoses, assigned by routine clinical evaluation at various treatment settings, are based on nationally applied operationalized diagnostic criteria and unbiased by hindsight at outcome. Diagnostic imprecisions likely exist in assessment of severity of depression, in poor recognition of delusional hopelessness of psychotic depression, and missed diagnoses of comorbid alcohol dependence. The previous and subsequent course of depression and clinical status at the time of death remain unknown. Therefore, our results presumably underestimate the actual effects. Bipolar disorder was probably underdiagnosed, and suicide attempts not requiring somatic hospitalization or occurring more than 4 years earlier remained unexamined. Uninvestigated risk factors include hopelessness, impulsive-aggressive tendencies or cluster B traits, and family history of suicide $(9,11)$. The socioeconomic status was susceptible to change after baseline, except for the highest level of education if attained. Despite the clearance period of 11-31 years some patients could have had previous hospitalizations longer time ago. However, based on the FHDR data, the number is likely to be very low because only $3 \%$ of consecutive admissions have an interval longer than 11 years and $0.5 \%$ longer than 21 years (median length of our clearance period). Psychiatric care represents treatment as usual with known regional differences (17). Possible effects of treatment and whether gender differences exist in provision of healthcare services or in treatment practices for depression remain unknown.

The long-term cumulative probability of suicide in men $(7.7 \%)$ and women $(3.9 \%)$ of this first-hospitalized cohort (8) is comparable to the findings of a similar Danish long-term study of first-treated depressive patients (7). The results on probability of suicide and age at time of suicide represent lowerboundary estimations because the cohort is not yet followed up throughout the lifetime. The nationallevel findings enable generalizability, whereas the cohort likely represents the most severe patients with depression. It remains unknown, to what extent risk factors for suicide between outpatient and inpatient populations diverge, or in this study varied over time along with the substantially declining risk for suicide (8). While prediction of suicide at individual level remains a difficult task, the findings may inform clinical practice and preventive efforts. 


\section{Aaltonen et al.}

\section{Acknowledgements}

We thank Marjut Grainger for data management and statistical assistance. The study was funded by the Helsinki University Hospital (research grants Y1070TKK14/1403, Y1012YTKK1/30101/1403, and TYH2016217), the Finnish Medical Foundation, and the Finnish Foundation for Psychiatric Research.

\section{Conflict of interest}

The authors declare no competing interests.

\section{References}

1. Сно SE, Na KS, Сно SJ, Im JS, Kang SG. Geographical and temporal variations in the prevalence of mental disorders in suicide: systematic review and meta-analysis. J Affect Disord 2016;190:704-713.

2. Qin P, Agerbo E, Mortensen PB. Suicide risk in relation to socioeconomic, demographic, psychiatric, and familial factors: a national register-based study of all suicides in Denmark, 1981-1997. Am J Psychiatry 2003;160:765-772.

3. Pirkola S, Sohlman B, Heila H, Wahlbeck K. Reductions in postdischarge suicide after deinstitutionalization and decentralization: a nationwide register study in Finland. Psychiatr Serv 2007;58:221-226.

4. Bostwick JM, Pankratz VS. Affective disorders and suicide risk: a reexamination. Am J Psychiatry 2000;157:1925-1932.

5. Chung DT, Ryan CJ, Hadzi-Pavlovic D, Singh SP, Stanton C, LARGe MM. Suicide rates after discharge from psychiatric facilities: a systematic review and meta-analysis. JAMA Psychiatry 2017;74:694-702.

6. Simon GE, VonKorfF M. Suicide mortality among patients treated for depression in an insured population. Am J Epidemiol 1998;147:155-160.

7. Nordentoft M, Mortensen PB, Pedersen CB. Absolute risk of suicide after first hospital contact in mental disorder. Arch Gen Psychiatry 2011;68:1058-1064.

8. Aaltonen KI, Isometsa E, Sund R, Pirkola S. Decline in suicide mortality after psychiatric hospitalization for depression in Finland between 1991 and 2014. World Psychiatry 2018;17:110-112.

9. Hawton K, Casanas I, Comabella C, Haw C, Saunders K. Risk factors for suicide in individuals with depression: a systematic review. J Affect Disord 2013;147:17-28.

10. Large M, Sharma S, Cannon E, Ryan C, Nielssen O. Risk factors for suicide within a year of discharge from psychiatric hospital: a systematic meta-analysis. Aust N Z J Psychiatry 2011;45:619-628.

11. Isometsa E. Suicidal behaviour in mood disorders-who, when, and why? Can J Psychiatry Revue 2014;59:120-130.

12. Arsenault-Lapierre G, Kim C, Turecki G. Psychiatric diagnoses in 3275 suicides: a meta-analysis. BMC Psychiatry 2004:4:37.

13. Hawton K. Sex and suicide. Gender differences in suicidal behaviour. Br J Psychiatry 2000;177:484-485.

14. Freeman A, Mergl R, Kohls E et al. A cross-national study on gender differences in suicide intent. BMC Psychiatry 2017; 17:234

15. Dalca IM, McGirr A, Renaud J, Turecki G. Gender-specific suicide risk factors: a case-control study of individuals with major depressive disorder. J Clin Psychiatry 2013;74:1209-1216.

16. Oquendo MA, Bongiovi-Garcia ME, Galfalvy H et al. Sex differences in clinical predictors of suicidal acts after major depression: a prospective study. Am J Psychiatry 2007; 164:134-141.

17. Pirkola S, Sund R, Sailas E, Wahlbeck K. Community mental-health services and suicide rate in Finland: a nationwide small-area analysis. Lancet 2009;373:147-153.

18. Sotkanet.fi statistical information on welfare and health in Finland [Internet]. National Institute for Health and Welfare. Available from: https://www.sotkanet.fi/sotkanet/en/ index?

19. Kuoppasalmi K, Lönnqvist J, Pylkkänen K, Huttunen M. Classification of mental disorders in Finland. A comparison of the Finnish classification of mental disorders in 1987 with DSM III-R. Psychiatria Fennica 1989;20: 65-81.

20. Sund R. Quality of the Finnish Hospital Discharge Register: a systematic review. Scand J Public Health 2012; 40:505-515

21. Isometsa E, Sund R, Pirkola S. Post-discharge suicides of inpatients with bipolar disorder in Finland. Bipolar Disord 2014;16:867-874

22. Lunetta P, Lounamaa A, Sinvonen S. Surveillance of injuryrelated deaths: medicolegal autopsy rates and trends in Finland. Injury Prev 2007;13:282-284.

23. Official Statistics of Finland (OSF). Quality Description: Causes of death 2014. Available from: http://www.stat.fi/ til/ksyyt/2014/ksyyt_2014_2015-12-30_laa_001_en.html.

24. Hoyer EH, Olesen AV, Mortensen PB. Suicide risk in patients hospitalised because of an affective disorder: a follow-up study, 1973-1993. J Affect Disord 2004;78:209217

25. Kessing LV. Severity of depressive episodes according to ICD-10: prediction of risk of relapse and suicide. Br J Psychiatry 2004;184:153-156.

26. Simon GE, Coleman KJ, Rossom RC et al. Risk of suicide attempt and suicide death following completion of the Patient Health Questionnaire depression module in community practice. J Clin Psychiatry 2016;77:221-227.

27. Rimimaki K, Vuorilehto M, Melartin T, HaukKa J, Isometsa E. Incidence and predictors of suicide attempts among primary-care patients with depressive disorders: a 5-year prospective study. Psychol Med 2014;44:291-302.

28. Leadholm AK, Rothschild AJ, Nielsen J, Bech P, OsterGAARD SD. Risk factors for suicide among 34,671 patients with psychotic and non-psychotic severe depression. J Affect Disord 2014;156:119-125.

29. Melartin TK, Rytsala HJ, Leskela US, Lestela-Mielonen PS, SOKERO TP, IsOMETSA ET. Severity and comorbidity predict episode duration and recurrence of DSM-IV major depressive disorder. J Clin Psychiatry 2004;65:810-819.

30. JaAskelainen E, Juola T, Korpela $\mathrm{H}$ et al. Epidemiology of psychotic depression-systematic review and meta-analysis. Psychol Med 2018;48:905-918.

31. Carroll R, Metcalfe C, Gunnell D. Hospital presenting self-harm and risk of fatal and non-fatal repetition: systematic review and meta-analysis. PLoS ONE 2014;9 e89944.

32. Olfson M, Wall M, Wang S, Crystal S, Gerhard T, Blanco C. Suicide following deliberate self-harm. Am J Psychiatry 2017; 174:765-774.

33. Fedyszyn IE, Erlangsen A, Huorthoj C, Madsen T, Nordentoft M. Repeated suicide attempts and suicide among individuals with a first emergency department contact for attempted suicide: a prospective, nationwide, Danish register-based study. J Clin Psychiatry 2016;77 832-840.

34. Isometsa ET, LonNQvist JK. Suicide attempts preceding completed suicide. Br J Psychiatry 1998;173:531-535. 
35. Bostwick JM, Pabbati C, Geske JR, McKean AJ. Suicide attempt as a risk factor for completed suicide: even more lethal than we knew. Am J Psychiatry 2016;173:1094 1100 .

36. Agerbo E, Gunnell D, Mortensen PB, Eriksson T, Qin P, WestergaARd-Nielsen N. Risk of suicide in relation to income level in people admitted to hospital with mental illness: nested case-control study. Commentary: Suicide and income-is the risk greater in rich people who develop serious mental illness? BMJ (Clinical research ed) 2001;322:334-335.

37. Qin P, Agerbo E, Westergard-Nielsen N, Eriksson T, MorTENSEN PB. Gender differences in risk factors for suicide in Denmark. Br J Psychiatry 2000;177:546-550.

38. Kessler RC, Bromet EJ. The epidemiology of depression across cultures. Annu Rev Public Health 2013;34:119-138.

39. Agerbo E. High income, employment, postgraduate education, and marriage: a suicidal cocktail among psychiatric patients. Arch Gen Psychiatry 2007;64:1377-1384.

40. Isometsa ET, Henriksson MM, Aro HM, Heikrinen ME, Kuoppasalmi KI, Lonnqvist JK. Suicide in major depression. Am J Psychiatry 1994;151:530-536.
41. Wang M, Alexanderson K, Runeson B, Mittendorfer-RutZ E. Sick-leave measures, socio-demographic factors and health care as risk indicators for suicidal behavior in patients with depressive disorders-a nationwide prospective cohort study in Sweden. J Affect Disord 2015;173:201-210.

42. Pirkola S, Isometsa E, Lonnqvist J. Do means matter?: Differences in characteristics of Finnish suicide completers using different methods. J Nervous Mental Dis 2003;191:745-750

\section{Supporting Information}

Additional Supporting Information may be found in the online version of this article:

Table S1. Baseline sociodemographic and clinical characteristics of patients at first lifetime psychiatric hospitalization for depression in Finland from 1991 to 2011

Table S2. Age-adjusted hazard ratios for suicide after first lifetime psychiatric hospitalization for depression followed for 628514 person-years 